Real-time Associations between Young Adults' Momentary Pain and Prescription Opioid Misuse Intentions in Daily Life

\author{
Lauren M. Papp \\ University of Wisconsin-Madison \\ Chrystyna D. Kouros \\ Southern Methodist University \\ John J. Curtin \\ University of Wisconsin-Madison
}

(C) 2020, American Psychological Association. This paper is not the copy of record and may not exactly replicate the final, authoritative version of the article. Please do not copy or cite without authors' permission. The final article will be available, upon publication, via its DOI: $10.1037 / a m p 0000648$

\begin{abstract}
Author Note
Lauren M. Papp, Department of Human Development and Family Studies, University of Wisconsin-Madison. Chrystyna D. Kouros, Department of Psychology, Southern Methodist University. John J. Curtin, Department of Psychology, University of Wisconsin-Madison.
\end{abstract}

We have no known conflict of interest to disclose. Research reported in this publication was supported by the National Institute on Drug Abuse of the National Institutes of Health under 
Award Number R01DA042093. The content is solely the responsibility of the authors and does not necessarily represent the official views of the National Institutes of Health. We gratefully acknowledge the UWellness Study's participants and research assistants, especially Alexandra Barringer and Shari Blumenstock.

Correspondence concerning this article should be addressed to Lauren M. Papp, Department of Human Development and Family Studies, University of Wisconsin-Madison, 1300 Linden Drive, Madison, WI 53706. E-mail: papp@wisc.edu 


\begin{abstract}
Managing pain has been identified (mainly through retrospective reports) as a robust motivator for individuals engaging in prescription opioid misuse. However, surprisingly little work has directly examined whether momentary pain experiences are associated with prescription opioid misuse in daily life. Participants included 297 young-adult college students recruited on the basis of recent prescription drug misuse. Ecological momentary assessment over a 28-day period was utilized to collect participants' pain experiences and prescription opioid misuse intention and behavior. Hierarchical generalized linear modeling and binary logistic regression tested hypotheses. Findings revealed that higher in-the-moment pain was positively associated with intentions to engage in prescription opioid misuse, accounting for report timing and participant sex and background substance use. Also, the between-person result indicated that participants who reported higher levels of pain across the reporting period were more likely to intend to misuse prescription opioids in daily life. There was a similar reliable association between higher pain ratings across the reporting period and greater likelihood of engaging in misuse behavior, although actual misuse frequency was low. Reliable moderation was observed: As hypothesized, the within-person association between momentary pain and misuse intentions was stronger for females compared to males. Also, the within-person link between pain and misuse intentions was stronger for those who reported lower (vs. higher) levels of problematic alcohol use; this was not consistent with the hypothesized direction. Understanding the role of college students' pain in their intentions to engage in prescription opioid misuse is important for informing future research and prevention efforts.
\end{abstract}

Keywords: ecological momentary assessment, young adults, pain, prescription drug misuse, substance use

Public significance statement: This study identified young-adult participants' pain experiences as a real-time predictor of intentions to misuse prescription opioids in daily life. The 
associations were stronger for females than males, and among participants who reported lower levels of problematic alcohol use. The results provide novel information about the pain - opioid misuse connection during an age period that sets the foundation for later adult health. 


\section{Real-time Associations between Young Adults' Momentary Pain and Prescription Opioid Misuse Intentions in Daily Life}

Prescription opioid misuse and abuse is a leading public health challenge (Jalal et al., 2018) and young adults are particularly affected (Tapscott \& Schepis, 2013). The misuse behavior itself is harmful to individuals and society and problematic for its established links with alcohol and other drug abuse, mental health problems, risky sexual behaviors, and overdose deaths (Ali et al., 2015; Benotsch, Koester, Luckman, Martin, \& Cejka, 2011; Harries, Lust, Christenson, Redden, \& Grant, 2018; McCabe, Cranford, \& Boyd, 2006; Webster, 2017). An extensive research base has identified subjective pain as a robust risk factor for prescription opioid misuse (Kaye et al., 2017; Voon, Karamouzian, \& Kerr, 2017). While support for this link has been found in epidemiological work in general population samples (Fischer \& Argento, 2012), the vast majority of the evidence is based on clinical-based samples of individuals with chronic pain or substance disorders (e.g., Jamison, Link, \& Marceau, 2009; Liebschutz et al., 2010), leaving a gap in our understanding of the pain - opioid misuse connection before the cycle of enduring pain and substance use problems is firmly established.

Also, despite broad recognition that pain is implicated in the current opioid epidemic (Carpenter, Lane, Bruehl, \& Trull, 2019; Chisholm-Burns, Spivey, Sherwin, Wheeler, \& Hohmeier, 2019; Kolodny et al., 2015; Wilkerson, Kim, Windsor, \& Mareiniss, 2016), virtually no evidence exists to show whether pain experienced by an individual in the moment is related to prescription opioid misuse intention or behavior. This is surprising, given successful application of ecological momentary assessment (EMA) to characterize risk factors for other substance use (e.g., tobacco, Berg et al., 2019; marijuana, Shrier, Walls, Kendall, \& Blood, 2012). The current study sought to address these gaps by investigating real-time associations between pain 
experiences and prescription opioid misuse intentions in daily life among male and female college students with elevated risk of prescription drug misuse. The college years during young adulthood reflect a particularly vulnerable point in the life course for experiencing substance problems (Schulenberg \& Maggs, 2002), and health-related functioning in this period has a unique and lasting impact on the quality of adult development (Brown et al., 2008). We tested background polysubstance use as a moderator to understand whether problematic alcohol or other drug use strengthens linkages between momentary pain and prescription opioid misuse; results can also clarify whether links found in daily life are accounted for by global substance tendencies.

Research aimed at understanding individuals' motivations, or reasons, for engaging in prescription opioid misuse has identified pain relief as a robust intention. An earlier survey of opioid misuse among adolescents (Boyd, McCabe, Cranford, \& Young, 2006) found that most respondents who misused pain medication in the past year reported doing so only for pain management, consistent with its medical indication. Brandt, Taverna, and Hallock (2014) studied prescription drug misuse behaviors and motivations via a survey administered to 303 students at a single college. Among the 110 participants who reported any prescription medication misuse in their lifetime, nearly one-third had a current prescription for prescription pain relievers and $11.8 \%(n=13)$ reported pain medication misuse in the past month. One-quarter of the participants who ever misused pain medications identified "self-medication" as the reason, and one-third reported mixing pain medications with other drugs, including marijuana, alcohol, or cocaine. Similarly, Lord, Brevard, and Budman (2011) conducted an online Facebook survey to understand college students' experiences with prescription drug misuse measured over the past year and the associated motivations. While the most common motivations reported for pain 
medication misuse were to relax, get high, and have fun, pain management was endorsed by nearly $20 \%$ of those who misused. Females were significantly more likely than males to report misusing opioids to help with ongoing pain. Moreover, the motive of managing pain was endorsed significantly more among those who engaged in regular misuse (once a month or more) as compared to those who reported infrequent misuse (Lord et al., 2011).

Recently, longitudinal tests spanning periods of years implicate pain as an antecedent factor for prescription opioid misuse across development. Drawing from the Add Health study, Groenewald, Law, Fisher, Beals-Erickson, and Palermo (2019) found that chronic pain experienced during adolescence reliably increased the likelihood of engaging in prescription opioid misuse in adulthood, after accounting for known covariates. In addition, McCabe and colleagues (2019) examined a national sample of late adolescents' (during the last year of high school) prescription opioid misuse characteristics (e.g., motives, frequency) and followed up at age 35. The majority of adolescents who engaged in past-year prescription opioid misuse reported occasional to frequent misuse along with non-pain relief motives and simultaneous use with alcohol or other drugs. Results from adjusted models indicated that adolescents who reported misusing opioids for pain relief had significantly greater likelihood of substance use disorder symptoms at age 35 than adolescents with no misuse history (McCabe et al., 2019). Yet another longitudinal study captured pain and opioid use disorder and found that early pain led to higher prevalence and incidence of prescription opioid use disorders over several years, whereas early opioid use disorder did not predict subsequent pain (Blanco et al., 2016). Together, these prospective studies showed that functioning in late adolescence sets a foundation for later adult problems, and that prescription opioid misuse precipitated by pain holds particular risk. 
While existing studies are informative for generating knowledge on pain as a contributing factor to engagement in prescription opioid misuse, they have generally relied on approaches that had participants identify their motivations retrospectively, or following the behavior of interest. A potential limitation of the findings, therefore, is that the motivation for the misuse behavior was captured after the respondent experienced any effect of the substance. This design presents a particular challenge in that the clinical effect of pain medication may bias participants' reports of their motivations. The recent longitudinal work (e.g., Groenewald et al., 2019) that identifies pain as a robust predictor of prescription opioid misuse over multiple years provides convincing evidence for testing pain experiences in the moment as more proximal risk factors of misuse, thereby accelerating the knowledge base and contributing to prevention and treatment efforts. A wealth of research focused on other substances has demonstrated the significance of identifying immediate antecedents or triggers of substance cravings or behaviors in real-time and naturalistic environments (e.g., Cleveland \& Harris, 2010; Kennedy, Epstein, Phillips, \& Preston, 2013). Therefore, we introduce a rigorous assessment of pain in the moment as a contextual correlate of prescription opioid misuse intention and behavior to strengthen the interpretation of any pain - opioid misuse linkages that emerge based on sampling in daily life.

Recent studies point to the utility of measuring pain experiences in daily life. For example, Sznitman, Baruch, Greene, and Gelkpf (2018) examined physical pain and cannabis use in daily life, testing between- and within-subject associations in a sample of 182 regular cannabis users. Contrary to the authors' hypotheses, elevated experiences of pain were not associated with cannabis use occasions at the within- or between-subject level. However, once individuals engaged in cannabis use, they tended to use larger amounts during instances when experiencing elevated levels of pain (Sznitman et al., 2018). In addition, Carpenter and 
colleagues (2019) utilized EMA to collect pain and opioid use among 34 chronic pain patients on opioid therapy and found that pain and negative affect were bidirectionally linked to opioid use. These initial studies show promising applications of EMA to capture pain and substance use in daily life and encourage extension to other samples of individuals with elevated risk for prescription drug misuse during pivotal developmental periods.

\section{The Current Study}

We implemented EMA to shed light on naturalistic experiences right before prescription drug misuse, minimize recall biases associated with substance behavior, and obtain contextual correlates of real-time misuse. Our design collected reports of intention to misuse 4 classes of prescriptions along with contextual factors and hypothesized predictors in a combination of signal- and event-contingent reporting. When intention to misuse any prescription class was endorsed, a brief follow-up prompt was sent 15 minutes later and participants indicated if misuse had recently occurred. The 15-minute window was selected on the basis of its use in EMA research that identified antecedents of young adults' smoking behavior (Thrul, Bühler, \& Ferguson, 2014).

The first question examined whether college students' pain experiences in daily life were associated with real-time prescription opioid misuse intention and behavior. In line with selfmedication theory (Khantzian, 1997), we hypothesized that individuals would be more likely to endorse prescription opioid misuse intention and behavior in moments of higher-than-normal levels of pain. Also, individuals who, on average, report higher levels of pain over the reporting period would be more likely to endorse prescription opioid misuse intention and behavior in daily life. Sex differences have been identified in prior work. Specifically, McCabe, Boyd, Cranford, and Teter (2009) conducted a large college-based survey of prescription drug misuse 
and motivations for misuse. They identified a self-treatment subtype ( $39 \%$ of the sample), or people who misused prescriptions for reasons consistent with the main indication of the drug, and found women were more likely characterized as self-treatment. Recently, however, Evans et al. (2017) conducted multivariate models using a sample of young adults recruited on the basis of prescription opioid misuse, and reported that greater physical pain was an independent predictor of frequent opioid misuse for males but not females. Acknowledging mixed results in the extant literature, we hypothesized that the within- and between-person associations would be stronger among females as compared to males.

The second question tested whether the pain-opioid misuse linkage is stronger at higher levels of background substance use. Consistent with polysubstance use models (Morley, Ferris, Winstock, \& Lynskey, 2017), research shows that young adults' substance behaviors go together such that those with higher alcohol and other drug use also are more likely to engage in prescription drug misuse (Garnier et al., 2009; McCauley et al., 2011; Miramontes et al., 2019; Schepis, Acheson, Zapp, \& Swartzwelder, 2019). Therefore, we predicted that individuals reporting higher levels of problematic alcohol and other drug use would report more prescription opioid misuse intentions in daily life. We also hypothesized that these individuals would evidence a stronger within-person link between their pain and prescription opioid misuse intentions in daily life.

\section{Method}

\section{Participants}

Between September 2017 and September 2019, participants at a large university in the mid-western U.S. were continuously enrolled into an ongoing longitudinal study on daily behaviors and health in college life. The current study is drawn from the first phase of the larger 
project. Participants were recruited via flyers and announcements (e.g., newspaper ads, emails to enrolled students) that stated, "We are particularly interested in how people use prescription medications." Prospective participants completed an online screening and a telephone call was scheduled to confirm eligibility. Inclusion criteria were (a) being enrolled as a freshman or sophomore; (b) being 18 to 21 years of age; and, for the risk sub-sample of participants included herein, (c) endorsing recent prescription drug misuse of one or more medication. We adapted McCabe's (2008) measure of yearly and lifetime prescription drug misuse to assess misuse in the past 3 months. Specifically, the screening measure assured confidential responses and described, "Sometimes people use prescription drugs in ways that a doctor did not direct them to. Please think back over the past 3 months and consider whether you have used the following types of medications in any way a doctor did not direct you to use them, including using it without a prescription of your own; using it in greater amounts, more often, or longer than you were told to take it; or using it in any way a doctor did not direct you to use it." This question was asked for 4 prescription medication classes with common examples listed, including pain relievers (e.g., OxyContin, Percocet, hydrocodone, codeine, morphine, fentanyl, Vicodin), tranquilizers (e.g., Xanax, Ativan, Valium, Klonopin), stimulants (e.g., Dexedrine and Adderall, Ritalin, Concerta), and sedatives or barbiturates (e.g., Ambien, Lunesta, Sonata). Response options were Yes/No and prospective participants could endorse misuse of multiple classes.

Of the 300 risk sub-sample participants enrolled into the project, most $(73.3 \%)$ endorsed recent misuse of one medication class; the remainder endorsed misuse of two or more classes. The majority of participants (77.3\%) reported recent misuse of prescription stimulants; the next highest class was pain medications ( $22.3 \%$ of the sample), followed by prescription tranquilizers (19.3\%), and prescription sedatives or barbiturates $(11.3 \%)$. These results are in line with 
national survey data showing college students have the highest prescription misuse rates for stimulants (Schepis, Teter, \& McCabe, 2018).

Two participants did not return for the second lab session or return their data collection devices, and EMA data from 1 participant was not retrievable due to a device malfunction. The sample thus consisted of 297 participants (69\% female) with an average age of 19.5 years $(S D=$ .71). Slightly more than half (56.6\%) were freshmen. In terms of racial and ethnic background, 83.2\% self-identified as White and $8.1 \%$ as Asian; nearly $7 \%$ reported Hispanic or Latino/a background. The remaining participants (8.4\%) self-identified as American Indian/Alaska Native, Black or African American, Native Hawaiian or Pacific Islander, or reported multiple or other races (individual identities included $<3 \%$ of respondents); one participant $(0.3 \%)$ did not respond. Participants were ethnically and racially representative of the campus from which they were sampled. Half of the sample $(50.5 \%)$ reported a pain prescription in their lifetime and $1.4 \%$ had a current prescription for pain medication.

\section{Procedure}

Prior to the study, university institutional review board approval and a National Institutes of Health Certificate of Confidentiality were obtained. Participants attended 2 lab sessions that were scheduled an average of 35 days apart and were trained to complete reporting procedures in daily life between the sessions. During the first session, participants completed informed consent procedures and survey measures and were trained to use an iPod Touch application designed specifically for the present research; they chose a private password to access the application and completed a sample report in the lab. Access to all other device features was restricted. The scheduled reporting period started the following day. Although the typical reporting period was scheduled for the 28 days following the first lab session, some participants continued reporting 
until they returned their device at the second lab session; reports obtained across all days were retained in the current analyses to maximize statistical power for hypotheses testing.

Consistent with published EMA protocols on addictions (Buckner et al., 2015; Cooney et al., 2007; Preston \& Epstein, 2011), both signal- and event-contingent assessments were administered. Signal-contingent reporting involved responding to a device prompt sent within four time windows (8:00 a.m.-11:30 a.m., 11:30 a.m.-3:00 p.m., 3:00 p.m.-7:00 p.m., and 7:00 p.m.-11:00 p.m.); prompts were sent at varying times within each window across days. Participants were instructed to respond as soon as possible as appropriate. Participants were also trained to self-initiate a report any time they intended to take a medication listed in any way a doctor did not direct them to use it (see Measures). Momentary report questions focused on participants' current location and social context and potential triggers of prescription drug misuse (e.g., mood states, pain, stressors, other substance behaviors). Signal- and event-based assessments were identical. A signal-contingent prompt was not sent within 2 hours after a selfinitiated report had been completed to reduce burden. If misuse intention of one or more of the medication classes was endorsed, participants were sent a brief follow-up 15 minutes later (Thrul et al., 2014) to assess misuse behaviors that might have occurred since the completion of the associated report; participants were instructed to respond to the follow-up within $15 \mathrm{~min}$. All time-stamps of report and follow-up completion were automatically recorded. During the second lab session, participants returned their devices and completed additional measures, including a modified Timeline Followback (TLFB; Robinson, Sobell, Sobell, \& Leo, 2014) adapted for prescription drug misuse. A research assistant then unlocked the device, downloaded the EMA reports to a secure server, and reset the devices. Participants received their choice of electronic or check payments; compensation included $\$ 75$ for session 1, \$84 for reporting in daily life 
(prorated for partial completion), \$55 for session 2, and a \$36 bonus incentive for achieving high compliance across the planned reporting period.

\section{Measures}

Prescription opioid misuse intention and behavior. Following Buckner and colleagues' (2015) EMA protocol for cannabis use in daily life, participants were asked on each momentary report, "Are you about to take a medication listed here in any way a doctor did not direct you to use it? Remember, this can include using a medication without a prescription of your own; using it in greater amounts, more often, or longer than you were told to take it; or using it in any other way a doctor did not direct you to use it." Participants indicated Yes or No for 4 classes of prescription drugs, adapted from McCabe (2008) (with examples provided): pain relievers, tranquilizers, stimulants, and sedatives or barbiturates. When participants responded Yes to one or more of the intention questions, they received a follow-up prompt 15 minutes after this report was completed. Participants were asked, "Have you recently taken a medication listed here, in any way a doctor did not direct you to use it?” The same 4 medication classes and examples were presented. This study used prescription opioid misuse intention from the momentary assessments and opioid misuse behavior from the follow-ups; responses to each item were recorded as $0(\mathrm{No})$ or $1($ Yes $)$.

Pain. Each momentary assessment prompted participants to, "Rate your current pain." The response set on this item ranged from 0 (no pain) to 10 (worst pain). The average pain rating for individuals across the reporting period was $1.23(S D=1.24$, range: $0-5.57)$, and the average maximum pain rating per participant across the reporting period was $5.31(S D=2.52$; range: 0 10). The intraclass correlation for pain ratings was 0.48 , indicating that $48 \%$ of the variance in momentary reports of pain was between-person and 52\% of the variability was within-person. 
Background substance use. Background substance use was measured during the first lab session and was comprised of problematic alcohol and other drug use. The Rutgers Alcohol Problem Index (RAPI; White \& Labouvie, 1989) consists of 18 items that examine various consequences of alcohol use that could have been experienced recently. Sample items include “went to work or school high or drunk" and "neglected your responsibilities." Following Neal, Corbin, and Fromme (2006), participants responded to each item using options of 0 (did not experience) or 1 (experienced at least one time in the last 3 months). Endorsed consequences were summed such that higher scores indicate greater alcohol use and alcohol-related consequences. The average RAPI score was $3.94(S D=3.31)$. In the current sample, $14.8 \%$ of participants had a score of 8 or above, suggesting the need for clinical treatment.

The 10-item Drug Abuse Screening Test (DAST-10; Skinner, 1982) is a measure of pastyear drug use. The measure's standard instructions have respondents consider non-medical use of prescription medications, along with drugs such as marijuana, cocaine, crack, meth, hallucinogens, and heroin; participants are instructed to exclude alcohol and tobacco from consideration. Given the current study's enrollment criteria, we modified the instructions by having participants exclude non-medical use of prescription medications (along with alcohol and tobacco) from consideration. All of the items refer to drug use in general. One item asks about any involvement with drugs other than those required for medical purposes. One item asks whether the respondent is always able to stop using drugs when they want to (reverse scored). The other items tap potential consequences of drug use; a sample question is, "Have you engaged in illegal activities in order to obtain drugs?" Response options are Yes/No. Endorsed items were summed with higher scores indicating higher drug use. Scores of 1-2 reflect potential drug 
problems and scores of 3 and above reflect moderate to severe drug problems. The average DAST-10 score was $2.38(S D=1.96)$. In the current sample, $36.7 \%$ had a score of 3 or above.

\section{Analytic Plan}

The analysis plan was preregistered at Open Science Framework (osf.io/ugazd). We used multilevel modeling to examine within-person associations between pain and prescription opioid misuse intentions. Specifically, models were run as a three-level hierarchical generalized model (HGLM) for Bernoulli outcomes to account for the nested data (i.e., moments nested within days, days nested within people) and binary dependent variable. Models were run using HLM v.8 (Raudenbush, Bryk, Cheong, \& Congdon, 2019). We followed centering guidelines for disentangling within-person from between-person associations (Bolger \& Laurenceau, 2013). A benefit of this approach is that HLM uses all available reports and draws from all respondents (whether or not they endorsed the dependent variable), providing more weight to those with more data and, therefore, more reliable estimates. Pain ratings at Level 1 were person-centered to represent the extent to which one's momentary pain level was higher or lower than their average pain level across the reporting period. The Level 1 model also included Moment, to account for any systematic effects of reporting moment of the day on intentions to misuse prescription pain medication. Level 2 accounted for Day; no day-level covariates were included. Individuals’ average pain level (i.e., averaged across EMA reports) was included as a covariate at Level 3 (grand-mean centered). Thus, analyses tested the extent to which within-person fluctuations in pain ratings predicted intentions to misuse prescription opioids in the same moment. Level 3 tested for between-person associations; that is, the extent to which individuals who experience more pain, on average, also report more prescription opioid misuse intention, on average. 
Following Chinn (2000), odds ratios were converted to Cohen's $d$ values to help interpret all effect sizes.

To test for sex differences in the within-person associations between pain and prescription opioid misuse intentions, sex was included at Level 3 as a predictor of the intercept (i.e., main effect of sex) along with the cross-level interaction with momentary pain (i.e., Sex X Pain interaction). To test the extent to which within-person associations between pain and prescription opioid misuse intentions differed based on individuals' background alcohol and other drug use, we included the RAPI and DAST-10 scores at Level 3 as predictors (in the same model) of the intercept (i.e., main effects of alcohol and other drug use) along with their crosslevel interactions with momentary pain (i.e., Alcohol x Pain interaction; Other Drug Use x Pain interaction).

\section{Results}

\section{Preliminary Results}

On average, participants each completed 80.45 reports $(S D=25.85$; range: $11-130)$ across 27.46 days $(S D=6.29$; range $=4-37)$, reflecting a $M$ completion rate of $69 \%$ of expected reports. Approximately one-half of the sample $(n=147 ; 49.5 \%)$ continued to provide reports after their scheduled 28-day reporting period had ended. These participants provided an average of 9.16 reports $(S D=4.77$; range $2-23)$, on an average of 2.54 days $(S D=1.59$; range $1-8)$, after the scheduled period; these were retained in analyses. The maximum number of momentary reports completed per day, on average, was $4.49(S D=1.09)$. Thus, together there were 23,651 total momentary reports completed by 297 participants; of these, there were 38 reports in which 22 participants indicated intentions to misuse an opioid prescription $(2.6 \%$ of reports included intentions to misuse any class of medication). Follow-up prompts were completed within 30 
minutes from 109 participants, yielding 303 follow-up reports from those who indicated they intended to misuse any class of medication. Of these 109 participants, $9.2 \%(n=10)$ indicated that they had misused an opioid prescription.

\section{Pain and Intentions to Misuse Prescription Opioids}

Results from the HGLM testing associations between pain and intentions to misuse prescription opioids are presented in Table 1 (Model 1). Moment was a significant positive predictor of the likelihood of intent to misuse prescription opioids, $O R=1.65,95 \% \mathrm{CI}[1.63$, 1.68], $p<.001$, indicating that the likelihood of endorsing intentions to misuse increased during the day. Over and above moment of the day, there was a positive within-person association between pain and intentions to misuse prescription opioid medication, $O R=1.16,95 \% \mathrm{CI}[1.14$, $1.18], p<.001$, indicating that higher pain than usual was associated with a greater likelihood of endorsing intention to misuse an opioid prescription in the moment (i.e., concurrently). A significant between-person association also emerged, $O R=1.22,95 \% \mathrm{CI}[1.19,1.25], p<.001$, and indicated that individuals who had higher levels of pain across the reporting period were more likely, on average, to report intention to misuse prescription opioids. Effect sizes for both the within- and between-person associations were small (see Table 1).

Sex differences. Results from models testing sex differences in associations between pain and intentions to misuse prescription opioids are in Table 1 (Model 2). In addition to the main effect of sex, $O R=1.54,95 \% \mathrm{CI}[1.43,1.66], p<.001$, indicating that males, on average, were more likely to report intending to misuse prescription opioid medication, there was a crosslevel interaction between sex and momentary pain ratings, $O R=0.86,95 \% \mathrm{CI}[0.84,0.89], p<$ .001. Results from a simple slopes analysis indicated that the within-person association between 
momentary pain and intention to misuse opioid medication was stronger for females, $O R=1.20$

$95 \%$ CI $[1.18,1.22], p<.001$, compared to males, $O R=1.09,95 \%$ CI $[1.06,1.11], p=.012$.

Problematic alcohol and other drug use. Results from models testing problematic alcohol and other drug use as moderators are presented in Table 1 (Model 3). Positive betweenperson main effects for both problematic alcohol, $O R=1.02,95 \%$ CI $[1.02,1.03], p<.001$, and other drug use, $O R=1.07,95 \%[1.05,1.08], p<.001$, were found, revealing that individuals who, on average, report greater problematic alcohol use, or greater problematic other drug use, were also more likely, on average, to report intending to misuse prescription opioids in daily life. Further, problematic alcohol use moderated the within-person association between pain and intention to misuse prescription medication; however, this was a small effect size and was not in the hypothesized direction, $O R=0.99,95 \%$ CI $[0.988,0.999], p=.023$. Simple slopes analysis of this interaction indicated that within-person fluctuation in pain, such that pain was higher than usual, was more strongly related to the likelihood of intending to misuse prescription opioids for those who reported lower levels of problematic alcohol use, $b=0.18, S E=0.01, p<.001$, as compared to those with higher levels of problematic alcohol use, $b=0.14, S E=0.01, p<.001$.

\section{Pain and Misuse of Prescription Opioids on the Follow-ups}

Given the low frequency of prescription opioid misuse, we did not have enough data points to run the intended HGLM model or to test the hypothesized moderators. As a post-hoc analysis, we ran a binary logistic regression to predict between-person associations between participants' average pain rating across the reporting period and the likelihood that they misused prescription opioids during the reporting period, accounting for participant sex and background substance use. Results indicated that higher levels of pain were associated with a greater 
likelihood of misusing prescription opioid medication in daily life; the effect size was small in magnitude (Table 2).

\section{Consistency in Participants' Reports of Prescription Opioid Misuse Across Methods}

To explicate our finding of lower frequencies for actual misuse relative to intentions to misuse, we explored the methodological possibility that some instances of misuse behavior occurred outside of the EMA follow-up window (i.e., within 30 minutes) and, therefore, were not captured by a subsequent report. We linked information obtained during EMA reporting and during the TLFB conducted in the second lab session. Results from this post-hoc analysis indicated reliable agreement in participants reporting that they had engaged in prescription opioid misuse behavior in daily life and when looking back over the reporting period, $\chi^{2}=$ 106.93, $d f=1, p<.001$. Specifically, prescription opioid misuse behavior was endorsed by 17 participants on the TLFB and 10 participants on the EMA, with 8 endorsing the behavior across both measures.

\section{Discussion}

Our study's EMA design captured participants' real-time pain experiences and opioid misuse intention and behavior among college students with elevated risk for prescription drug misuse. Findings revealed that higher in-the-moment pain was positively associated with intentions to engage in prescription opioid misuse, accounting for timing of the reports. In addition, participants who reported higher levels of pain across the reporting period were more likely to intend to misuse prescription opioids in daily life. Although low frequencies required us to examine misuse behavior using a between-subject approach, we similarly found a reliable association between higher pain ratings across the reporting period and greater likelihood of engaging in prescription pain misuse in daily life. The assessment of pain experiences and 
intentions to misuse pain medications in daily life represents an improvement over the available studies that measured motivations for misuse after the substance behavior had occurred.

It remains unclear why we found lower frequencies for behavior reports relative to intentions. Given our primary interest in collecting hypothesized predictive factors before an instance of prescription drug misuse occurred, we trained participants to respond to EMA prompts throughout the day and to self-initiate when misuse was about to occur. We also intentionally had participants use a separate study device (with restricted access to other features) to make sure reports of sensitive behaviors could not be connected to identifying information stored on their own devices. It is possible that by the time participants engaged in the misuse behavior, their reporting device was no longer accessible. Nonetheless, results from a comparison of participant reports of misuse behavior across TLFB and EMA approaches documented reliable consistency in the two, thereby strengthening confidence in the overall findings based on reporting in daily life.

As with other substance use research, it is also possible that social desirability concerns or measurement reactivity led to under-reporting. We plan to undertake additional research to investigate these possibilities in depth. Notably, we conducted an initial post-hoc analysis and found that EMA reporting day and moment number across the study were not reliably associated with participants endorsing prescription pain medication intention or behavior (all $p$-values $>$ .12), suggesting minimal reactivity to reporting in daily life. Nevertheless, acknowledging that our EMA approach did not capture all instances of prescription opioid misuse that occurred in daily life, we view the collection of rich assessments of momentary pain experiences and prescription opioid misuse intentions using highly secure procedures as benefits of the design. 
Some moderating differences emerged. In terms of participant sex, whereas males were significantly more likely than females to intend to misuse prescription opioids in daily life, females showed a stronger positive within-person link between momentary pain and prescription opioid misuse intention. Background substance use was also tested. Consistent with polysubstance use models, people reporting higher problematic alcohol and other drug use were more likely to intend to misuse opioids in daily life. Problematic alcohol use moderated the within-person association between pain and misuse intention such that those with higher alcohol use were less likely to misuse prescription opioids at moments of relatively higher pain. Although not what we originally expected, the findings can be interpreted in the context of other recent research. Votaw, McHugh, and Witkiewitz (2019) conducted a latent class analysis based on data from the 2015 National Survey on Drug Use and Health. They found that among adults with past-year opioid misuse, one-quarter had co-occurring alcohol use disorder (AUD). Interestingly, pain relief was the most common motive (vs. recreational or mixed motives) for recent opioid misuse, endorsed by $56.1 \%$ of the sample. However, individuals with AUD were significantly less likely to be in the pain relief class, suggesting alcohol and opioid misuse are not utilized together to self-medicate pain (Votaw et al., 2019). In other words, to the extent a person is drinking more alcohol, in general, they may be less likely to need pain medication to self-treat pain or other symptoms of distress.

\section{Clinical Considerations}

Using community-sampled college students, the current study tested the pain - opioid misuse linkage that is overwhelmingly demonstrated in patient-based populations. As one example, Alford and colleagues (2016) found that adult primary care patients who report prescription drug misuse or illicit drug use tend to exhibit chronic pain and pain-related 
dysfunction. A significant portion of their sample engaged in heavy drinking to self-treat pain, thereby contributing to a cycle of enduring pain and substance use problems. The current study contributes to research that is needed to shed light on risk factors to assist with modifying experiences prior to the development of the challenging interplay between pain and prescription opioid misuse and abuse. A next step in this line of investigation, therefore, could be to extend the method described here to young adults who experience an injury, migraines, or other condition that places them at risk for developing chronic pain (Graham \& Streitel, 2010) and potentially displaying more frequent problematic opioid use behaviors (Edlund et al., 2014). Future work should also be designed to understand the mechanisms (e.g., early exposure to opioids, family adversity) that underlie variability in risk for prescription opioid misuse (intention and behavior) to manage pain during young adulthood.

Results from our between-subject tests revealing that individuals with greater background problematic substance use are more likely to intend to misuse opioid prescriptions in daily life also offer clinical implications. According to the U.S. Food \& Drug Administration, alcohol use is not recommended when taking prescription pain medications (Esser, Guy, Zhang, \& Brewer, 2019; Ives et al., 2006). From a prevention perspective, college students with access to pain prescriptions, and those with elevated likelihood of opioid misuse (based on recent prescription behavior) who endorse problematic drinking or other drug use, should be advised of the risks.

\section{Study Limitations}

The findings should be interpreted while considering several limitations. One limitation is that the method of reporting in daily life captured a low frequency of prescription opioid misuse. The low base rates could reflect recent changes in awareness and availability of prescription medications; opioid prescribing practices, in general, have declined since 2010 in response to the 
ongoing epidemic (Levy, Paulozzi, Mack, \& Jones, 2015). Nevertheless, our results indicated reliable associations between in-the-moment pain and prescription opioid misuse intention in daily life. It is evident from the longitudinal findings linking earlier pain to later substance use disorders that additional insight into how this process unfolds is urgently needed to continue advancing public health needs (Jalal et al., 2018). Also, the effect sizes were small in magnitude; replication is needed to bolster clinical interpretation of the results. Another potential limitation is that our measures of substance use, RAPI and DAST-10, had different time anchors in their instructions (i.e., past 3 months and past year, respectively), which is not ideal. However, the scales were selected based on their use and validation in similar samples, and thus allow for meaningful comparisons to other published work.

In closing, the current study suggests that helping college students who struggle with pain throughout the day may reduce their risk for pain medication misuse intention, and, therefore, addresses serious medication practices during a developmental period that holds enduring implications for adult health (Stroud, Walker, Davis, \& Irwin, 2015). 


\section{References}

Alford, D. P., German, J. S., Samet, J. H., Cheng, D. M., Lloyd-Travaglini, C. A., \& Saitz, R. (2016). Primary care patients with drug use report chronic pain and self-medicate with alcohol and other drugs. Journal of General Internal Medicine, 31, 486-491. doi:10.1007/s11606-016-3586-5

Ali, M. M., Dean, Jr., D., Lipani, R., Dowd, W. N., Aldridge, A. P., \& Novak, S. P. (2015). The mental health consequences of nonmedical prescription drug use among adolescents. Journal of Mental Health Policy and Economics, 18, 3-15.

Benotsch, E. G., Koester, S., Luckman, D., Martin, A. M., \& Cejka, A. (2011). Non-medical use of prescription drugs and sexual risk behavior in young adults. Addictive Behaviors, 36, 152-155. doi:10.106/j.addbeh.2010.08.027

Berg, C. J., Haardörfer, R., Payne, J. B., Getachew, B., Vu, M., Guttentag, A., \& Kirchner, T. R. (2019). Ecological momentary assessment of various tobacco product use among young adults. Addictive Behaviors, 92, 38-46. doi:10.106/j.addbeh.2018.12.014

Blanco, C., Wall, M. M., Okuda, M., Wang, S., Iza, M., \& Olfson, M. (2016). Pain as a predictor of opioid use disorder in a nationally representative sample. The American Journal of Psychiatry, 173, 1189-1195. doi:10.1176/appi.ajp.2016.15091179

Bolger, N., \& Laurenceau, J.-P. (2013). Intensive longitudinal methods: An introduction to diary and experience sampling research. New York: Guilford Press.

Boyd, C. J., McCabe, S. E., Cranford, J. A., \& Young, A. (2006). Adolescents' motivations to abuse prescription medications. Pediatrics, 118, 2472-2480. doi:10.1542/peds.2006-1644

Brandt, S. A., Taverna, E. C., \& Hallock, R. M. (2014). A survey of nonmedical use of tranquilizers, stimulants, and pain relievers among college students: Patterns of use 
among users and factors related to abstinence in non-users. Drug and Alcohol Dependence, 143, 272-276. doi:10.1016/j.drugalcdep.2014.07.034

Brown, S. A., McGue, M., Maggs, J. L., Schulenberg, J., Hingson, R., Swartzwelder, S., ...Murphy, S. (2008). A developmental perspective on alcohol and youths 16 to 20 years of age. Pediatrics, 121, S290-S310. doi:10.1542/peds.2007-2243D

Buckner, J. D., Zvolensky, M. J., Crosby, R. D., Wonderlich, S. A., Ecker, A. H., \& Richter, A. (2015). Antecedents and consequences of cannabis use among racially diverse cannabis users: An analysis from Ecological Momentary Assessment. Drug and Alcohol Dependence, 147, 20-25. doi:10.106/j.drugalcdep.2014.12.022

Carpenter, R. W., Lane, S. P., Bruehl, S., \& Trull, T. J. (2019). Concurrent and lagged associations of prescription opioid use with pain and negative affect in daily lives of chronic pain patients. Journal of Consulting and Clinical Psychology, 87, 872-886. doi:10.1037/ccp0000402

Chinn, S. (2000). A simple method for converting an odds ratio to effect size use in metaanalysis. Statistics in Medicine, 19, 3127-3131. doi:10.1002/10970258(20001130)19:22<3127::AID-SIM784>3.0.CO;2-M

Chisholm-Burns, M. A., Spivey, C. A., Sherwin, E., Wheeler, J., \& Hohmeier, K. (2019). The opioid crisis: Origins, trends, policies, and the roles of pharmacists. American Journal of Health-System Pharmacy, 76, 424-435. doi:10.1093/ajhp/zxy089

Cleveland, H. H., \& Harris, K. S. (2010). The role of coping in moderating within-day associations between negative triggers and substance use cravings: A daily diary investigation. Addictive Behaviors, 35, 60-63. doi:10.1016/j.addbeh.2009.08.010 
Cooney, N. L., Litt, M. D., Cooney, J. L., Pilkey, D. T., Steinberg, H. R., \& Oncken, C. A. (2007). Alcohol and tobacco cessation in alcohol-dependent smokers: Analysis of realtime reports. Psychology of Addictive Behaviors, 21, 277-286. doi:10.1037/0893164X.21.3.277

Edlund, M. J., Martin, B. C., Russo, J. E., DeVries, A., Braden, J. B., \& Sullivan, M. D. (2014). The role of opioid prescription in incident opioid abuse and dependence among individuals with chronic noncancer pain: The role of opioid prescription. The Clinical Journal of Pain, 30, 557-564. doi:10.1097/AJP.0000000000000021

Esser, M. B., Guy, G. P., Zhang, K., \& Brewer, R. D. (2019). Binge drinking and prescription opioid misuse in the U.S., 2012-2014. American Journal of Preventive Medicine, 57, 197-208. doi:10.106/j.amepre.2019.02.025

Evans, T. I., Liebling, E. J., Green, T. C., Hadland, S. E., Clark, M. A., \& Marshall, B. D. L. (2017). Associations between physical pain, pain management, and frequency of nonmedical prescription opioid use among young adults: A sex-specific analysis. Journal of Addictive Medicine, 11, 266-272. doi:10.197/ADM.0000000000000318

Fischer, B., \& Argento, E. (2012). Prescription opioid related misuse, harms, diversion and interventions in Canada: A review. Pain Physician, 15(3 Suppl), ES191-203.

Garnier, L. M., Arria, A. M., Caldiera, K. M., Vincent, K. B., O’Grady, K. E., \& Wish. E. D. (2009). Nonmedical analgesic use and concurrent alcohol consumption among college students. The American Journal of Drug and Alcohol Abuse, 35, 334-338. doi:10.1080/00952990903075059 
Graham, J. E., \& Streitel, K. L. (2010). Sleep quality and acute pain severity among young adults with and without chronic pain: The role of biobehavioral factors. Journal of Behavioral Medicine, 33, 335-345. doi:10.1007/s10865-010-9263-y

Groenewald, C. B., Law, E. F., Fisher, E., Beals-Erickson, S. E., \& Palermo, T. M. (2019). Associations between adolescent chronic pain and prescription opioid misuse in adulthood. Journal of Pain, 20, 28-37. doi:10.1016/j.pain.2018.07.007

Harries, M. D., Lust, K., Christenson, G. A., Redden, S. A., \& Grant, J. E. (2018). Prescription opioid medication misuse among university students. The American Journal on Addictions, 27, 618-624. doi:10.1111/ajad.12807

Ives, T. J., Chelminski, P. R., Hammett-Stabler, C. A., Malone, R. M., Perhac, J. S., Potisek, N. M., Shilliday, B. B., DeWalt, D. A., \& Pignone, M. P. (2006). Predictors of opioid misuse in patients with chronic pain: A prospective cohort study. BMC Health Services Research, 6, 46. doi:10.1185/1472-6963-6-46

Jalal, H., Buchanich, J. M., Roberts, M. S., Balmert, L. C., Zhang, K., \& Burke, D. S. (2018). Changing dynamics of the drug overdose epidemic in the United States from 1979 through 2016. Science, 361, p. eaau1184. doi:10.1126/science.aau1184

Jamison, R. N., Link, C. L., \& Marceau, L. D. (2009). Do pain patients at high risk for substance misuse experience more pain?: A longitudinal outcomes study. Pain Medicine, 10, 10841094. doi:10.1111/j.1526-4637.2009.00679.x

Kaye, A. D., Jones, M. R., Kaye, A. M., Ripoll, J. G., Galan, V., Beakley, B. D., ...Manchikanti, L. (2017). Prescription opioid abuse in chronic pain: An updated review of opioid abuse predictors and strategies to curb opioid abuse: Part 1. Pain Physician, 20(2S), S93-S109. doi:10.36076/ppj.2017.s111 
Kennedy, A. P., Epstein, D. H., Phillips, K. A., \& Preston, K. L. (2013). Sex differences in cocaine/heroin users: Drug-use triggers and craving in daily life. Drug and Alcohol Dependence, 132, 29-37. doi:10.1016/j.drugalcdep.2012.12.025

Khantzian, E. J. (1997). The self-medication hypothesis of substance use disorders: A reconsideration of recent applications. Harvard Review of Psychiatry, 4, 231-244. doi:10.3109/10673229709030550

Kolodny, A., Courtwright, D. T., Hwang, C. S., Kreiner, P., Eadie, J. L., Clark, T. W., \& Alexander, G. C. (2015). The prescription opioid and heroin crisis: A public health approach to an epidemic of addiction. Annual Review of Public Health, 36, 559-574. doi:10.1146/annurev-publhealth-031914-122957

Levy, B., Paulozzi, L., Mack, K. A., \& Jones, C. M. (2015). Trends in opioid analgesicprescribing rates by specialty, U.S., 2007-2012. American Journal of Preventive Medicine, 49, 409-413. doi:10.106/j.amepre.2015.02.020

Liebschutz, J. M., Saitz, R., Weiss, R. D., Averbuch, T., Schwartz, S., Meltzer, E. C., ...Samet, J. H. (2010). Clinical factors associated with prescription drug use disorder in urban primary care patients with chronic pain. The Journal of Pain, 11, 1047-1055. doi:10.106/j/pain.2009.10.012

Lord, S., Brevard, J., \& Budman, S. (2011). Connecting to young adults: An online social network survey of beliefs and attitudes associated with prescription opioid misuse among college students. Substance Use \& Misuse, 46, 66-76.

doi:10.3109/10826084.2011.521371 
McCabe, S. E. (2008). Screening for drug abuse among medical and nonmedical users of prescription drugs in a probability sample of college students. Archives of Pediatrics \& Adolescent Medicine, 162, 225-231. doi:10.1001/archpediatrics.2007.41

McCabe, S. E., Boyd, C. J., Cranford, J. A., \& Teter, C. J. (2009). Motives for nonmedical use of prescription opioids among high school seniors in the United States. Archives of Pediatric Adolescent Medicine, 163, 739-744. doi:10.1001/archpediatrics.2009.120

McCabe, S. E., Cranford, J. A., \& Boyd, C. J. (2006). The relationship between past-year drinking behaviors and nonmedical use of prescription drugs: Prevalence of cooccurrence in a national sample. Drug and Alcohol Dependence, 84, 281-288. doi:10.1016/j.drugalcdep.2006.03.006

McCabe, S. E., Veliz, P. T., Boyd, C. J., Schepis, T. S., McCabe, V. V., \& Schulenberg, J. E. (2019). A prospective study of nonmedical use of prescription opioids during adolescence and subsequent substance use disorder symptoms in early midlife. Drug and Alcohol Dependence, 194, 377-385. doi:10.1016/j.drugalcdep.2018.10.027

McCauley, J. L., Amstadter, A. B., Macdonald, A., Danielson, C. K., Ruggerio, K. J., Resnick, H. S., \& Kilpatrick, D. G. (2011). Non-medical use of prescription drugs in a national sample of college women. Addictive Behaviors, 36, 690-695. doi:10.106/j.addbeh.2011.01.020

Miramontes, A. B., Moure-Rodríguez, L., Díaz-Geada, A., Rodríguez-Holguín, S., Corral, M., Cadaveira, F., \& Caamaño-Isorna, F. (2019). Heavy drinking and non-medical use of prescription drugs among university students: A 9-year follow-up. International Journal of Environmental Research and Public Health, 16, 2939. doi:10.3390/ijerph16162939 
Morley, K. I., Ferris, J. A., Winstock, A. R., \& Lynskey, M. T. (2017). Polysubstance use and misuse or abuse of prescription opioid analgesics: A multi-level analysis of international data. Pain, 158, 1138-1144. doi:10.1097.j.pain.0000000000000892

Neal, D. J., Corbin, W. R., \& Fromme, K. (2006). Measurement of alcohol-related consequences among high school and college students: Application of item response models to the Rutgers Alcohol Problem Index. Psychological Assessment, 18, 402-414. doi:10.1037/1040-3590.18.4.402

Preston, K. L., \& Epstein, D. H. (2011). Stress in the daily lives of cocaine and heroin users: Relationship to mood, craving, relapse triggers, and cocaine use. Psychopharmacology, 218, 29-37. doi:10.1007/s00213-011-2183-x

Raudenbush, S. W., Bryk, A. S., Cheong, Y. F., \& Congdon, R. (2019). HLM 8 for Windows [Computer software]. Skokie, IL: Scientific Software International, Inc.

Robinson, S. M., Sobell, L. C., Sobell, M. B., \& Leo, G. I. (2014). Reliability of the Timeline Followback for cocaine, cannabis, and cigarette use. Psychology of Addictive Behaviors, 28, 154-162. doi:10.1037/a0030992

Schepis, T. S., Acheson, S., Zapp, D., \& Swartzwelder, H. S. (2019). Alcohol use and consequences in matriculating US college students by prescription stimulant/opioid nonmedical misuse status. Addictive Behaviors, 98. Advance online publication. doi:10.1016/j.addbeh.2019.06.015

Schepis, T. S., Teter, C. J., \& McCabe, S. E. (2018). Prescription drug use, misuse and related substance use disorder symptoms vary by educational status and attainment in U.S. adolescents and young adults. Drug and Alcohol Dependence, 189, 172-177. doi:10.1016/j.drugalcdep.2018.08.017 
Schulenberg, J. E. \& Maggs, J. L. (2002). A developmental perspective on alcohol use and heavy drinking during adolescence and the transition to young adulthood. Journal of Studies on Alcohol, Supplement, s14, 54-70. doi:10.15288/jsas.2002.s14.54

Shrier, L. A., Walls, C. E., Kendall, A. D., \& Blood, E. A. (2012). The context of desire to use marijuana: Momentary assessment of young people who frequently use marijuana. Psychology of Addictive Behaviors, 26, 821-829. doi:10.1037/a0029197

Skinner, H. (1982). The Drug Abuse Screening Test. Addictive Behaviors, 7, 363-371. doi:10.1016/0306-4603(82)90005-3

Stroud, C., Walker, L. R., Davis, M., \& Irwin, Jr., C. E. (2015). Investing in the health and wellbeing of young adults. Journal of Adolescent Health, 56, 127-129. doi:10.106/j.adolhealth.2014.11.012

Sznitman, S., Baruch, Y. B., Greene, T., \& Gelkpf, M. (2018). The association between physical pain and cannabis use in daily life: An experience sampling method. Drug and Alcohol Dependence, 191, 294-299. doi:10.1016/j.drugalcdep.2018.07.014

Tapscott, B. E., \& Schepis, T. S. (2013). Nonmedical use of prescription medications in young adults. Adolescent Medicine, 24, 597-610.

Thrul, J., Bühler, A., \& Ferguson, S. G. (2014). Situational and mood factors associated with smoking in young adult light and heavy smokers. Drug and Alcohol Review, 33, 420-427. doi:10.1111/dar.12164

Voon, P., Karamouzian, M., \& Kerr, T. (2017). Chronic pain and opioid misuse: A review of reviews. Substance Abuse Treatment, Prevention, and Policy, 15, 36. doi:10.1186/s13011-017-0120-7 
Votaw, V. R., McHugh, K., \& Witkiewitz, K. (2019). Alcohol use disorder and motives for prescription opioid misuse: A latent class analysis. Substance Use \& Misuse, 54, 15581568. doi:10.1080/10826084.2019.1594904

Webster, L. R. (2017). Risk factors for opioid-use disorder and overdose. Anesthesia \& Analgesia, 125, 1741-1748. doi:10.1213/ANE.0000000000002496

White, H. R., \& Labouvie, E. W. (1989). Towards the assessment of adolescent problem drinking. Journal of Studies on Alcohol, 50, 30-37. doi:10.15288/jsa.1989.50.30

Wilkerson, R. G., Kim, H. K., Windsor, T. A., \& Mareiniss, D. P. (2016). The opioid epidemic in the United States. Emergency Medicine Clinics, 34, e1-e23. doi:10.1016/j.emc.2015.11.002 
Table 1

Within-person and Between-person Associations between Momentary Pain Ratings and

Prescription Opioid Misuse Intentions

\begin{tabular}{|c|c|c|c|c|}
\hline & $O R$ & $p$ value & $95 \% \mathrm{CI}$ & $d^{\mathrm{a}}$ \\
\hline \multicolumn{5}{|l|}{ Model 1} \\
\hline Intercept & 0.01 & $<.001$ & {$[0.008,0.009]$} & -- \\
\hline Person-Mean Pain Level (between-person) & 1.22 & $<.001$ & {$[1.19,1.25]$} & 0.11 \\
\hline Moment (within-person) & 1.65 & $<.001$ & {$[1.63,1.68]$} & 0.28 \\
\hline Momentary Pain LevelPC (within-person) & 1.16 & $<.001$ & {$[1.14,1.18]$} & 0.08 \\
\hline \multicolumn{5}{|l|}{ Model 2} \\
\hline Intercept & 0.01 & $<.001$ & {$[0.009,0.01]$} & -- \\
\hline $\operatorname{Sex}(0=$ female and $1=$ male $)($ between-person $)$ & 1.31 & $<.001$ & {$[1.25,1.36]$} & 0.15 \\
\hline Person-Mean Pain Level (between-person) & 1.20 & $<.001$ & {$[1.18,1.23]$} & 0.10 \\
\hline Moment (within-person) & 1.55 & $<.001$ & {$[1.53,1.57]$} & 0.24 \\
\hline Momentary Pain LevelPC (within-person) & 1.20 & $<.001$ & {$[1.18,1.22]$} & 0.10 \\
\hline Sex x Momentary Pain Level $\mathrm{PC}$ & 0.86 & $<.001$ & {$[0.84,0.89]$} & 0.08 \\
\hline \multicolumn{5}{|l|}{ Model 3} \\
\hline Intercept & 0.01 & $<.001$ & {$[0.008,0.009]$} & -- \\
\hline Problematic Alcohol Use (between-person) & 1.02 & $<.001$ & {$[1.02,1.03]$} & 0.01 \\
\hline Problematic Other Drug Use (between-person) & 1.07 & $<.001$ & {$[1.05,1.08]$} & 0.04 \\
\hline Person-Mean Pain Level (between-person) & 1.20 & $<.001$ & {$[1.17,1.23]$} & 0.10 \\
\hline Moment (within-person) & 1.65 & $<.001$ & {$[1.62,1.67]$} & 0.28 \\
\hline Momentary Pain LevelPC (within-person) & 1.17 & $<.001$ & {$[1.15,1.19]$} & 0.09 \\
\hline Alcohol x Momentary Pain LevelPC (within-person) & 0.99 & .023 & {$[0.988,0.999]$} & 0.004 \\
\hline Other Drug x Momentary Pain LevelPC (within-person) & 0.99 & .30 & {$[0.99,1.01]$} & 0.003 \\
\hline
\end{tabular}

Note. Results from population-average models with robust standard errors presented. $\mathrm{PC}=$

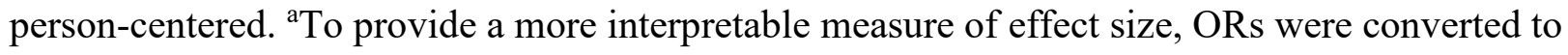
Cohen's $d$, using the formula provided by Chinn (2000): $\ln (\mathrm{OR}) *(\sqrt{3} / \pi)$. The absolute value for $d$ is presented when $O R<1.0$. 
Table 2

Results from Binary Logistic Regression Predicting Prescription Opioid Misuse Occurring during the Reporting Period

\begin{tabular}{lcccc}
\hline & OR & $p$ value & $95 \%$ CI & $d^{\mathrm{a}}$ \\
\cline { 2 - 5 } Intercept & 0.03 & $<.001$ & -- & 0.25 \\
Average Pain Rating across reporting period & 1.57 & .003 & {$[1.17,2.11]$} & 0.63 \\
Sex $(0=$ female and $1=$ male) & 3.12 & .17 & {$[0.62,15.83]$} & 0.01 \\
Problematic Alcohol Use & 1.01 & .90 & {$[0.83,1.24]$} & 0.01 \\
Problematic Other Drug Use & 1.28 & .16 & {$[0.91,1.79]$} & 0.14
\end{tabular}

Note. $N=109$ participants who completed a follow-up report. ${ }^{\text {a }}$ To provide a more interpretable measure of effect size, ORs were converted to Cohen's $d$, using the formula provided by Chinn (2000): $\ln (\mathrm{OR}) *(\sqrt{3} / \pi)$ 